\title{
A Variational Framework for Active and Adaptative Segmentation of Vector Valued Images
}

Mikaël Rousson — Rachid Deriche

$\mathbf{N}^{\circ} 4515$

July 2002

THÈME 3 



\title{
A Variational Framework for Active and Adaptative Segmentation of Vector Valued Images
}

\author{
Mikaël Rousson, Rachid Deriche \\ Thème 3 - Interaction homme-machine, \\ images, données, connaissances \\ Projet Odyssee \\ Rapport de recherche $\mathrm{n}^{\circ} 4515$ - July 2002 - 18 pages
}

\begin{abstract}
During the last few years, many efforts have been done in integrating different informations in a variational framework to segment images. Recent works on curve propagation were able to incorporate stochastic informations [14, 10] and prior knowledge on shapes [3,11]. The information inserted in these studies is most of the time extracted offline. Meanwhile, other approaches have proposed to extract region information during the segmentation process itself $[2,4,13]$.

Following these new approaches and extending the work in [10] to vector-valued images, we propose in this paper an entirely variational framework to approach the segmentation problem. Both, the image partition and the statistical parameters for each region are unkown.

After a brief reminder on recent segmenting methods, we will present a variational formulation obtained from a bayesian model. After that, we will show two different differentiations driving to the same evolution equations. Detailed studies on gray and color images of the 2-phase case will follow. And we will finish on an application to tracking which shows benefits of our dynamical framework.
\end{abstract}

Key-words: Level Set Theory, Color Image Segmentation, Adaptative Image Segmentation, Shape Gradient. 


\section{Une Approche Variationnelle pour une Segmentation Active et Adaptative d'Images Vectorielles}

Résumé : Si l'on s'intéresse aux derniers travaux sur la segmentation d'images, on s'aperçoit qu'un grand nombre d'entre eux consiste à incorporer diverses informations dans une approche variationnelle. Ainsi, de récentes études issues des contours actifs ont permis d'introduire des informations stochastiques $[14,10]$ et d'intégrer des à priori sur la forme des contours [8,3,11]. D'autres approches, considérées comme adaptatives, proposent d'extraire des informations régions durant le processus de segmentation $[2,4,13]$.

Dans ce papier, nous proposons une approche variationnelle qui généralise les travaux présentés dans [10] aux images couleur tout en estimant de manière adaptative les informations régions. Ainsi il n'est plus nécessaire d'avoir recours à des méthodes de clustering pour extraire les informations régions avant la segmentation.

Apres un bref rappel sur les méthodes de segmentations récentes, nous introduisons une formulation variationnelle obtenue à partir d'un modèle bayésien. Ensuite, deux types de différentiations sont présentées; les deux conduisant aux mêmes équations d'évolution. Nous montrons de nombreux résultats pour la segmentation en deux régions d'images en niveaux de gris et d'images couleurs. Les bénéfices apportés par le caractère adaptatif de la méthode sont clairement illustrés dans le cas du tracking d'un objet isolé. Enfin, nous étudions l'extension de la méthode pour la segmentation d'images en plus de deux régions.

Mots-clés : Théorie des courbes de niveaux, segmentation d'images couleurs, segmentation adaptative d'images, gradient de formes. 


\section{Introduction}

Considering the last studies in the domain, a variational formulation seems adequate to tackle the problem of segmenting images. In particular, this approach has shown its ability to integrate various cues in a common framework. Hence, different kind of informations can be used as direct constraint on the boundaries (regularity, tension, high gradient, shape prior) or indirect constraint induced by modules accounting for region integrity (homogeneity[2], texture classification [9], prior on spatial intensity distributions $[7], \ldots)$.

In most of the cases, a preprocessing step is necessary to extract the relevant information from the image before the partitioning process itself. Different types of informations can be extracted: the creation of an edge image can provide boundary informations, data clustering may be useful to determine a simple region module, complex filtering can help in extracting texture characteristics...

In this paper, no prior extraction of information is made. An entirely variational formulation is proposed, where region cues extraction is made jointly with the partitioning process. Recent related works can be found. In [2], the authors propose to use the mean to distinguish the regions, the mean of the regions being dynamically estimated during the evolution of the curve which delimits the regions. They generalize their model to deal with vector-valued data.

In [4], the criteria used to separate two smooth regions is their entropy, this one depending again on the moving border position. In [13], the authors have also proposed similar approaches. Bimodal images are segmented using Gaussian distributions with fixed variances and adaptative means. More recently, a non-parametric method using information theory have been presented in [5].

The method proposed in the next section can be seen as a generalization of the $C V$ model [2]. Actually, a more general variational formulation is obtained from the maximization of the a posteriori segmentation probability given an observed data. First, limiting our study to the 2-phase case, we will present two different ways of minimizing the functional driving both to the same evolution equations. One use a modified energy by an early introduction of level set functions, whereas the other method consists in differentiating directly the functional using recent results on shape derivation. Next, we will show some experimental results on gray-valued and color images, and also on sequences of images. Then, we will finish by making some comments on the limitations of the method and the possible extensions to an arbitrary number of regions.

RR $\mathrm{n}^{\circ} 4515$ 


\section{The segmentation problem}

Segmenting images consists of finding a partition of an observed data $I$ into homogeneous regions. These regions are characterized by statistical properties which represent a visual consistency. The interface between the regions is supposed to be regular.

Let $\Omega \in \mathbb{R}^{2}$ be open and bounded, and let $I: \Omega \rightarrow \mathbb{R}^{p}$ be the observed data. Let $\mathcal{P}(\Omega)$ be a partition of the domain and $\partial \Omega$ be the boundaries between the regions. We make the following assumptions: 1) $I$ is composed by a maximum of $N$ regions $\Omega_{i}$ verifying an hypothesis $\left.h_{i}, 2\right)$ the interface between the regions $\partial \Omega$ is regular.

Let $p_{\Theta_{i}}(I(x))$ be the conditional probability density function of a given value $I(x)$ with respect to the hypothesis $h_{i}$. The good segmentation of a given observed data respecting the hypotheses is obtained by solving the optimization problem with respect to the a posteriori segmentation probability, given the observation set: $p(P(\Omega) \mid I)$. Following the Geodesic Active Regions [10], we can reformulate the problem in terms of energy. Only two assumptions are made in [10]: all the partitions are equally possible and the pixels within each region are independent. Since these assumptions are reasonable in our case, the optimal frame partition is obtained by minimizing the energy:

$$
F(\Sigma, \mu, \partial \Omega)=\sum_{i=1}^{N} \int_{\Omega_{i}}-\log p_{\Theta_{i}}(I) d x+\operatorname{length}(\partial \Omega)
$$

To describe the visual consistency of a region, we need to define a family of density function. The choice of this family must be done such that it can represent/approximate the information of each region and it should be able to discriminate two different regions. For smooth non-textured images, a common choice is to use Gaussian distributions. It means that the conditional probability with respect to $h_{i}$ for a value $I$ is:

$$
p_{\theta_{i}}(I)=p_{\Sigma_{i}, \mu_{i}}(I)=\frac{1}{(2 \pi)^{\frac{p}{2}}\left|\Sigma_{i}\right|^{1 / 2}} e^{-\frac{1}{2}\left(I-\mu_{i}\right)^{T} \Sigma_{i}^{-1}\left(I-\mu_{i}\right)}
$$

Remark : As we will see on some experiments, the use of Gaussian densities may be also efficient on particular textured images. Actually, these images can be segmented by fitting Gaussian models even if these models do not give a good estimation of the region properties. 
Considering this family of distribution, we can rewrite the energy:

$$
\begin{aligned}
F(\Sigma, \mu, \partial \Omega)= & \frac{1}{2} \sum_{i=1}^{N} \int_{\Omega_{i}}\left(p \log (2 \pi)+\log \left|\Sigma_{i}\right|\right. \\
& \left.+\left(I-\mu_{i}\right)^{T} \Sigma_{i}^{-1}(I-\mu)\right) d x+\operatorname{length}(\partial \Omega)
\end{aligned}
$$

Such an energy has already been studied in [6] by looking for the minimum length description and in [14] with a region completion scheme. The first one is a variant of graduated non-convexity and the second one propose to use snakes in a region completion scheme. This last method solve the problem by iterating two steps: 1) estimate statistical parameters with fixed boundaries, 2) move the boundaries with constant statistical parameters.

Here, we propose a global variational framework using the level set theory. The energy minimum is obtained via a gradient descent with respect to the statistical parameters and the boundary position.

\section{On finding the minima}

We study two different approaches driving to a local minima of the functional (2) when considering the 2-phase problem for smooth, non-textured images. The energy corresponding to this particular case is:

$$
F(\partial \Omega, \Theta)=\int_{\Omega_{1}} e_{1}(x) \mathrm{d} x+\int_{\Omega_{2}} e_{2}(x) \mathrm{d} x+\operatorname{length}(\partial \Omega)
$$

where $\Theta=\left(\Sigma_{1}, \mu_{1}, \Sigma_{2}, \mu_{2}\right)$ and $e_{i}(x)=\log \left|\Sigma_{i}\right|+\left(I(x)-\mu_{i}\right)^{T} \Sigma_{i}^{-1}\left(I(x)-\mu_{i}\right)$.

Remark : Active contours without edges for vector-valued images presented in [2] can be seen as a particular case of this formulation where $\Sigma_{1}$ and $\Sigma_{2}$ are set to the identity matrix.

\subsection{First approach: extension of the integral terms to all the do- main}

A difficulty encountered when we want to derive (3) is the dependence with respect to the border position of the integration domains $\Omega_{1}$ and $\Omega_{2}$. As it has been done in the past [2], we extend these integrals to all the domain by using the level set

$\mathrm{RR} \mathrm{n}^{\circ} 4515$ 
function $\phi: \Omega \rightarrow \mathbb{R}$ defined as:

$$
\begin{cases}\phi(x)=\mathcal{D}(x, \partial \Omega), & \text { if } x \in \Omega_{1} \\ \phi(x)=-\mathcal{D}(x, \partial \Omega), & \text { if } x \in \Omega_{2}\end{cases}
$$

Using the same regularized form $H_{\epsilon}(z)$ of the heaviside function as in [2], the functional (3) can be written as:

$$
\begin{aligned}
E(\phi, \Theta)= & \int_{\Omega}\left(e_{1}(x) H_{\epsilon}(\phi)+e_{2}(x)\left(1-H_{\epsilon}(\phi)\right)\right) \mathrm{d} x \\
& +\operatorname{length}(\partial \Omega)
\end{aligned}
$$

The length term can also be expressed with respect to $\phi$ :

$$
\operatorname{length}(\partial \Omega)=\int_{\Omega}\left|\nabla H_{\epsilon}(\phi(x))\right| \mathrm{d} x
$$

The Euler-Lagrange equations obtained for the Gaussian parameters $\mu_{i}$ and $\Sigma_{i}$ can be directly solved. The solution gives expressions of $\mu_{i}$ and $\Sigma_{i}$ with respect to the level set function $\phi$ :

$$
\left\{\begin{array}{l}
\mu_{i}(\phi)=\frac{\int_{\Omega}\left(\mu_{i}-I(x)\right) \chi_{i}(\phi(x)) d x}{\int_{\Omega} \chi_{i}(\phi(x)) d x} \\
\Sigma_{i}(\phi)=\frac{\int_{\Omega}\left(\mu_{i}-I(x)\right)\left(\mu_{i}-I(x)\right)^{T} \chi_{i}(\phi(x)) d x}{\int_{\Omega} \chi_{i}(\phi(x)) d x}
\end{array}\right.
$$

where $\chi_{1}(\phi)=H_{\epsilon}(\phi)$ and $\chi_{2}(\phi)=1-H_{\epsilon}(\phi)$. These expressions are the estimation of the Gaussian parameters into the respective region which is coherent with the formulation of the problem.

Hence the energy depends only on $\phi$ :

$$
E(\phi, \Theta)=E(\phi, \Theta(\phi))=G(\phi)
$$

Then, it is possible to compute the first variations of $G$ with respect to $\phi$. A detailed description of the derivation can be found in annex. Using the result of the annex, we can write the following evolution equation for $\phi$ :

$$
\phi_{t}(x)=\delta_{\epsilon}(\phi(x))\left[\nu \cdot \operatorname{div}\left(\frac{\nabla \phi}{|\nabla \phi|}\right)+e_{2}(x)-e_{1}(x)\right]
$$

with $\delta_{\epsilon}(\phi)=H_{\epsilon}^{\prime}(\phi)$.

The implementation is straightforward: the level set function is evolved with a gradient descent using the equation (6) while the Gaussian parameters are updated at each iteration with respect to (5). 


\subsection{Second approach: direct derivation using shape derivation prin- ciple}

We can wonder how the use of a regularized function in the energy modifies our objective function. Using the shape derivative tool introduced in [1], it is possible to differentiate directly the functional (3).

We suppose that the statistical parameters are obtained using an estimation on the respective region as in (5):

$$
\left\{\begin{array}{l}
\mu_{i}\left(\Omega_{i}\right)=\frac{\int_{\Omega_{i}}\left(\mu_{i}-I(x)\right) d x}{\int_{\Omega_{i}} d x} \\
\Sigma_{i}\left(\Omega_{i}\right)=\frac{\int_{\Omega_{i}}\left(\mu_{i}-I(x)\right)\left(\mu_{i}-I(x)\right)^{T} d x}{\int_{\Omega_{i}} d x}
\end{array}\right.
$$

Then the functional depends only on the border position $\partial \Omega$ :

$$
E(\partial \Omega)=\int_{\Omega_{1}} e_{1}\left(x, \Omega_{1}\right) d x+\int_{\Omega_{2}} e_{2}\left(x, \Omega_{2}\right) d x+\nu \int_{\partial \Omega} \partial \dot{\Omega}(x) d x
$$

The integrals $D\left(\Omega_{i}\right)=\int_{\Omega_{i}} e_{i}\left(x, \Omega_{i}\right) d x$ may be differentiated with respect to the position of $\Omega_{i}$ using shape derivative. Concerning our case, the derivative gives a simple term (see annex):

$$
<D^{\prime}\left(\Omega_{i}\right), V>=+/-\int_{\partial \Omega} e_{i}\left(x, \Omega_{i}\right)(V(x) \cdot N(x)) d a(x)
$$

where $N(x)$ is the unit normal vector to $\partial \Omega$ at point $x$. The sign depends on the direction of $N$, i.e. if $\Omega_{i}$ is the inside region, the sign is minus otherwise it is positive. Now we can write the new evolving equation:

$$
\partial \Omega(x)_{t}=\left(e_{2}(x)-e_{1}(x)+\nu \kappa\right) \mathbf{N}
$$

where $\kappa$ is the curvature of the curve $(\partial \Omega)$.

It is efficient to use the level set theory to implement this equation. The level set function is defined as the distance function like in (3) and we get the following level set evolution equation:

$$
\phi_{t}(x)=\left[e_{2}(x)-e_{1}(x)+\nu \operatorname{div}\left(\frac{\nabla \phi}{|\nabla \phi|}\right)\right]|\nabla \phi|
$$

and the equation (6) is obtained: 1 ) by approximating $|\nabla \phi|$ by 1 since $\phi$ should be the distance function; 2) by introducing $\delta_{\alpha}(x)$ to impose the Narrow Band hypothesis: only local pixels have influence on the curve propagation.

$\mathrm{RR} \mathrm{n}^{\circ} 4515$ 

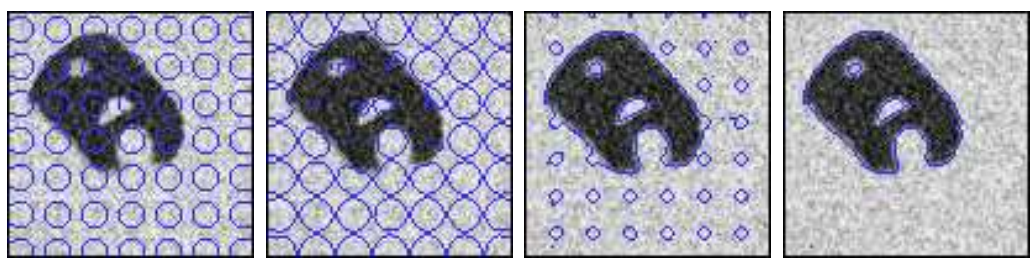

Figure 1: Two regions with different means - Contour evolution
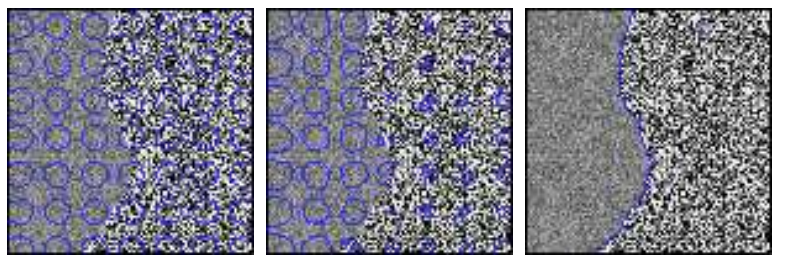

Figure 2: Two regions with different variances - Contour evolution

\section{Experiments}

The complexity of the variational method presented in the last section is high. The unknown parameters for region information can be up to 9 per region for color images ( 6 for the covariance matrix and 3 more for the mean).

\subsection{Gray-valued images}

For scalar images, the complexity is smaller. Only two parameters (mean and variance) are necessary to represent a region. The simplified evolving equation for this case is:

$$
\begin{aligned}
\phi_{t}(x)= & \delta_{\epsilon}(\phi(x))\left(\nu \cdot \operatorname{div}\left(\frac{\nabla \phi}{|\nabla \phi|}\right)\right. \\
& \left.+\log \frac{\sigma_{2}^{2}}{\sigma_{1}^{2}}-\frac{\left(I(x)-\mu_{1}\right)^{2}}{\sigma_{1}^{2}}+\frac{\left(I(x)-\mu_{2}\right)^{2}}{\sigma_{2}^{2}}\right)
\end{aligned}
$$

We show results on two synthetical images. The first one is composed by two regions with different means but same variances [Fig.1] while the second one has two regions different only by their variances [Fig.2]. 

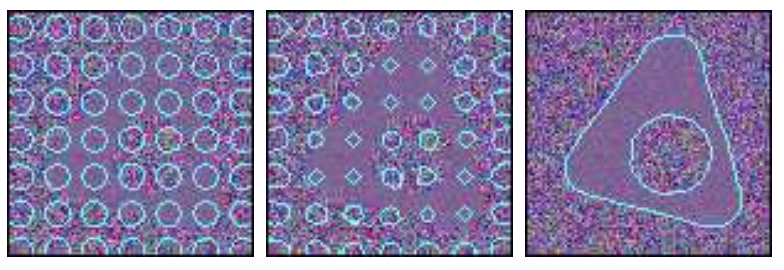

$$
\begin{gathered}
\mu_{1}=\mu_{2} \\
\Sigma_{1}=\left(\begin{array}{ccc}
\alpha_{1} & 0 & 0 \\
0 & \beta_{1} & 0 \\
0 & 0 & \gamma_{1}
\end{array}\right) \text { and } \Sigma_{1}=\left(\begin{array}{ccc}
\alpha & a_{1} & b_{1} \\
b_{1} & \beta & c_{1} \\
c_{1} & b_{1} & \gamma
\end{array}\right)
\end{gathered}
$$
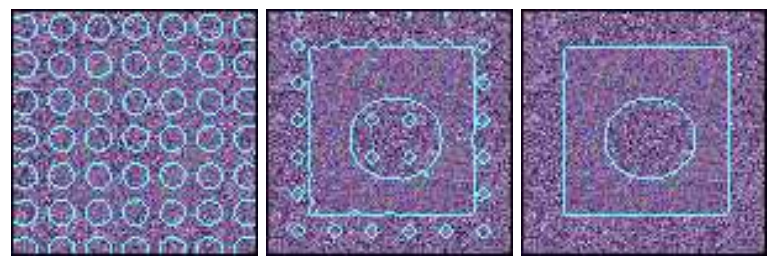

$$
\Sigma_{2}=\left(\begin{array}{ccc}
\alpha_{2} & 0 & 0 \\
0 & \beta_{2} & 0 \\
0 & 0 & \gamma_{2}
\end{array}\right) \text { and } \Sigma_{2}=\left(\begin{array}{ccc}
\alpha & a_{2} & b_{2} \\
b_{2} & \beta & c_{2} \\
c_{2} & b_{2} & \gamma
\end{array}\right)
$$

Figure 3: Synthetic test for color images - Contour evolution

\subsection{Color images}

Concerning color images, the complexity is higher but images composed by complex regions can be considered. First, we show results on synthetical data where 3-dimensional Gaussian models were used to generate data for each region. In both examples, the regions have the same mean but their covariance matrix is different. For the first example, auto-correlation coefficients (between color components) are different while only cross-correlation coefficients are different for the second example. Contrary to more classical approaches as the one presented in [2], our method is able to capture these differences [Fig.3].

Experiments on real images gave interesting results. Let us precise that we used the CIE-Lab color space such that distance between 3D points corresponds to perceptual color difference.

The first test consists in segmenting a hand photography placed on different backgrounds. Three backgrounds are considered: uniform (hue close to the hand's) [Fig.4(a)], noisy [Fig.4(b)] and textured [Fig.4(c)]. The algorithm succeeded in giving the expected partitioning for each test. Two more tests on natural images are shown. First, the "squirrel" image [Fig.??]: the regions are textured but they have 

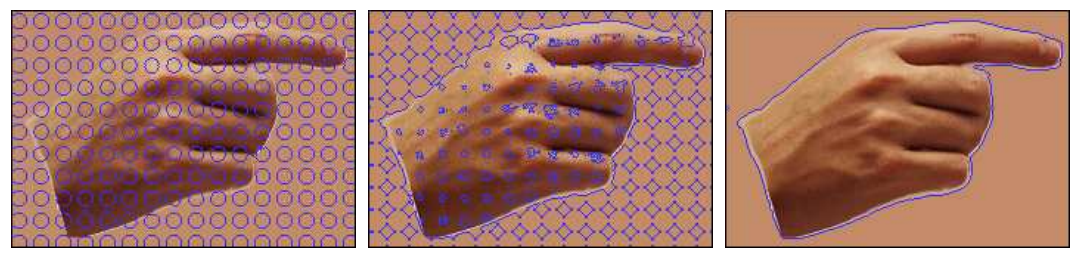

(a) Uniform background - Contour evolution
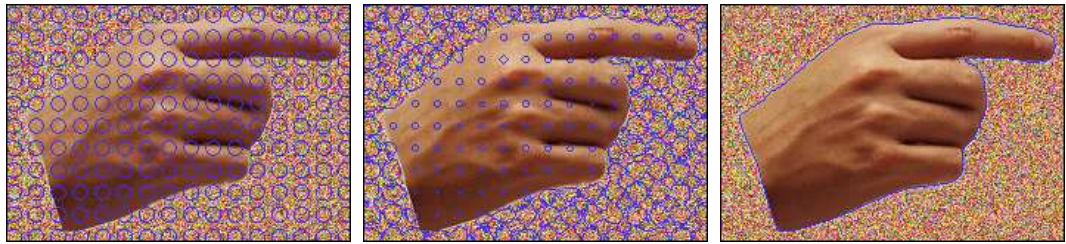

(b) Noisy background - Contour evolution
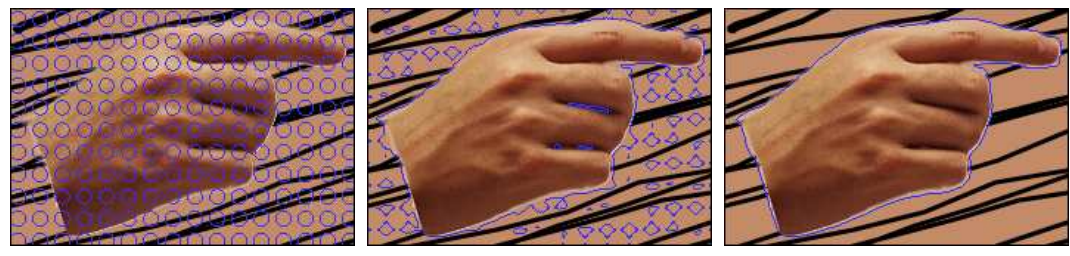

(c) Textured background - Contour evolution

Figure 4: "Hand" image (left: initial contour, center: evolving contour, right: final segmentation)

a really different color. The partitioning result looks very accurate [Fig.??]. Second example, the "Rocks on mars" image: the rock is slightly darker than the sand with a different texture. The obtained result is still satisfactory [Fig.6].

\subsection{Color image sequences}

The dynamical property of our method can be useful to segment a sequence of images. The hand examples presented here is composed by a moving object (a hand) placed 

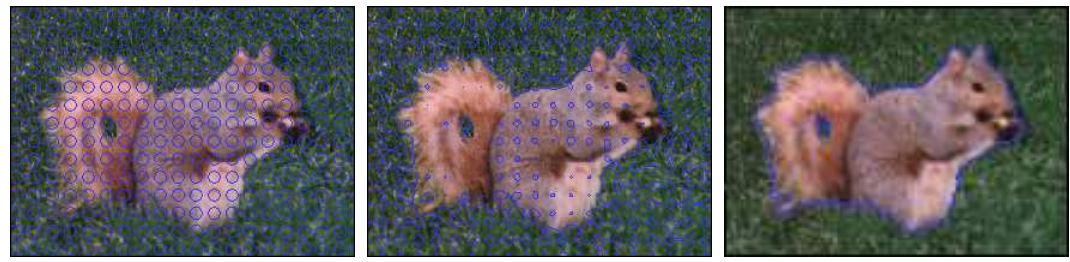

Figure 5: "Squirrel" image - Contour evolution
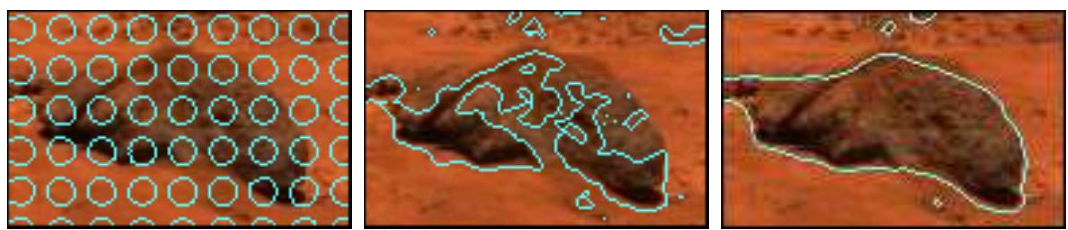

Figure 6: "Rocks on mars" image - Contour evolution
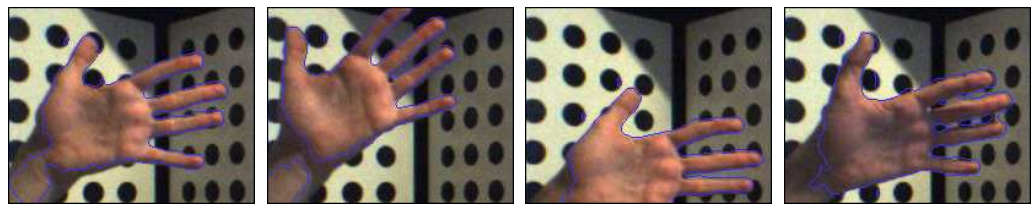

Figure 7: Hand sequence

in front of a textured background. Since the hand motion is composed by various rotations and translations, the luminosity of the object varies very much during the sequence. The result of the previous frame is used as initialization for the next frame. The method does not integrate additional terms based on motion properties as optical flow. Hence, the method is not limited to small displacements. As you can see on the example, the algorithm can naturally integrate luminosity modifications thanks to the dynamical Gaussian parameters. We can also remark that a Gaussian approximation for the background is not good at all but it is sufficient to differentiate it from the hand 


\subsection{Implementation remarks}

For all the tests, except the sequence, the same initialization with tiny circles was used. Such an initialization has been already used in the past. The reasons why we chose this initialization was its ability to detect easily holes and the convergence speed. However, other initializations have been tested such as a single circle. It still gives good results but the convergence speed decreases significantly. Moreover, we have to set only two parameters: the time step and the regularization weight $\nu$. For all the color examples, we used the same values: $d t=1$ and $\nu=1$.

Concerning the speed, we use an explicit discretization in time, then the evolution must be done slowly to be stable. On 100x100 images, only few seconds are required for scalar images while color images need around twenty seconds on a $1 \mathrm{Ghz}$ CPU. It seems possible to get close to real time using a semi-implicit scheme as the AOS [12]. To deal with larger images, we are in the process to develop a multi-scale approach.

\section{Generalization to $N$ regions}

We start from the functional (1) and we would like to extend each integral over $\Omega_{i}$ to all the image domain $\Omega$. We must find characteristic functions $\left\{\chi_{i}, i=1, . ., N\right\}$ such that:

$$
\begin{cases}\phi_{i}(x)>0 & \text { if } \mathrm{x} \in \Omega_{i} \\ \phi_{i}(x)=0 & \text { if } \mathrm{x} \in \partial \Omega_{i} \\ \phi_{i}(x)<0 & \text { otherwise }\end{cases}
$$

Actually, two different kind of characteristic functions have been proposed for this problem. One can simply associate one level set per region but pixels can be associated to multiple regions or to no region. An additional coupling term must be added to avoid that. A second way of defining $\chi_{i}$ has been propose in [2] by associating a characteristic function to each combination of level set signs. Hence only $\log (N)$ level sets are needed to segment an image into $N$ regions and each pixel is associated to one and only one region. For example, an image can be segmented in 4 regions using 2 level sets $\phi_{1}$ and $\phi_{2}$ by minimizing the following functional:

$$
\begin{aligned}
E\left(\phi_{1}, \phi_{2}, \Theta\right)= & \sum_{i=1}^{4} \int_{\Omega}-\log p_{\theta_{i}}(I(x)) \chi_{i}\left(\phi_{1}, \phi_{2}\right) d x \\
& +\operatorname{length}\left(\partial \Omega_{1} \cup \partial \Omega_{2}\right)
\end{aligned}
$$



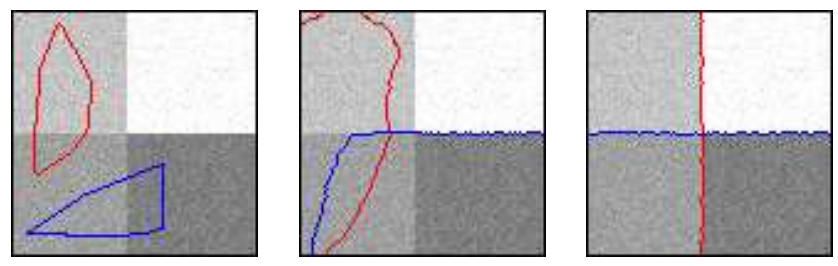

Figure 8: Four different means - Contour evolution

$$
\text { with }\left\{\begin{array}{l}
\chi_{1}\left(\phi_{1}, \phi_{2}\right)=H_{\epsilon}\left(\phi_{1}\right) H_{\epsilon}\left(\phi_{2}\right), \\
\chi_{2}\left(\phi_{1}, \phi_{2}\right)=\left(1-H_{\epsilon}\left(\phi_{1}\right)\right) H_{\epsilon}\left(\phi_{2}\right), \\
\chi_{3}\left(\phi_{1}, \phi_{2}\right)=H_{\epsilon}\left(\phi_{1}\right)\left(1-H_{\epsilon}\left(\phi_{2}\right)\right), \\
\chi_{4}\left(\phi_{1}, \phi_{2}\right)=\left(1-H_{\epsilon}\left(\phi_{1}\right)\right)\left(1-H_{\epsilon}\left(\phi_{2}\right)\right)
\end{array}\right.
$$

The derivation of (9) is similar to one for the 2-phase case and two evolution equations are obtained for $\phi_{1}$ and $\phi_{2}$. Making the notations $e_{i}(x)=\log \left|\Sigma_{i}\right|+(I(x)-$ $\left.\mu_{i}\right)^{T} \Sigma_{i}^{-1}\left(I(x)-\mu_{i}\right)$ and $H_{i}(x)=H_{\epsilon}\left(\phi_{i}(x)\right)$, the energy can be simply expressed as follow:

$$
\begin{aligned}
E\left(\phi_{1}, \phi_{2}\right)= & \int_{\Omega}\left(\left|H_{\epsilon}\left(\phi_{1}(x)\right)\right|+\left|H_{\epsilon}\left(\phi_{2}(x)\right)\right|\right) d x \\
& +\int_{\Omega}\left(e 1 H_{1} H_{2}+e_{2}\left(1-H_{1}\right) H_{2}+e_{3} H_{1}\left(1-H_{2}\right)+e_{4}\left(1-H_{1}\right)\left(1-H_{2}\right)\right) d x
\end{aligned}
$$

and the corresponding evolution equations for $\phi_{1}$ and $\phi_{2}$ are:

$$
\left\{\begin{array}{l}
\frac{\partial \phi_{1}}{\partial t}(x)=\delta_{\epsilon}\left(\phi_{1}(x)\right) \\
\frac{\partial \phi_{2}}{\partial t}(x)=\delta_{\epsilon}\left(\phi_{2}(x)\right)
\end{array}\left(\nu \cdot \operatorname{div}\left(\frac{\nabla \phi_{1}}{\mid \nabla \phi_{1}}\right)+\left(e_{1}-e_{2}\right) H_{2}+\left(e_{3}-e_{4}\right)\left(1-H_{2}\right)\right)\right.
$$

A nice example is shown [Fig.8] but the method becomes more sensible to the initial contour position on more complicated images. Moreover, the complexity becomes very high when the number of regions increase.

\section{Conclusion and future works}

We have presented a totally dynamical framework to segment scalar or vector-valued images. Two different approaches have been considered to tackle the problem of energy minimization, both drives to the same evolution equations. Convincing results 
were shown on synthetical and real images. The method looks particularly powerful in segmenting various type of images. A simple multi-dimensional Gaussian to represent each region is often sufficient to discriminate complex regions. Regarding the tracking example, improvements can be done by using for instance priors on the shape [11] or by integrating a more complex model for the background and a skin model for the hand. Moreover, we used here a parametric model assuming that probability densities of each region are close to Gaussian distributions. The development of non-parametric methods can be useful when dealing with textures which are characterized by moments of higher order. We are currently concentrating on these last issues to improve and generalize the method.

\section{A Derivation details}

\section{A.1 Energy extended over all the domain $\Omega$}

To show how the Euler-Lagrange equations are obtained, let us differentiate the following functional:

$$
\begin{aligned}
F(\phi) & =-\int_{\Omega} \log p(I(x) \mid \mu, \Sigma) \chi(\phi) d x \\
& =\frac{1}{2} \int_{\Omega}\left(\log |\Sigma|+(I(x)-\mu)^{T} \Sigma^{-1}(I(x)-\mu)\right) \chi(\phi) d x
\end{aligned}
$$

where $p(x \mid \mu, \Sigma)$ is the Gaussian density function with mean $\mu$ and covariance matrix $\Sigma(\mu$ and $\Sigma$ depend on $\phi(5))$

We compute the first variation of $F$ :

$$
\begin{aligned}
\left.\frac{\partial F(\phi+\epsilon \psi)}{\partial \epsilon}\right|_{\epsilon=0}= & \frac{1}{2} \int_{\Omega} \log p(I(x) \mid \mu, \Sigma) \chi^{\prime}(\phi) d x \\
& +\left.\frac{1}{2} \int_{\Omega} \frac{\partial G(x, \phi+\epsilon \psi)}{\partial \epsilon}\right|_{\epsilon=0} \chi(\phi) d x
\end{aligned}
$$

where $\left.G(x, \phi+\epsilon \psi)=\log |\Sigma(\phi+\epsilon \psi)|+(I(x)-\mu(\phi+\epsilon \psi))^{T} \Sigma(\phi+\epsilon \psi)^{-1}(I(x)-\mu(\phi+\epsilon \psi))\right)$ We can decompose the derivative of $G$ :

$$
\frac{\partial G(x, \phi+\epsilon \psi)}{\partial \epsilon}=\frac{\partial G}{\partial \mu} \frac{\partial \mu(\phi+\epsilon \psi)}{\partial \epsilon}+\frac{\partial G}{\partial \Sigma} \frac{\partial \Sigma(\phi+\epsilon \psi)}{\partial \epsilon}
$$

First we compute the gradient with respect to the mean vector $\mu$ :

$$
\frac{\partial G}{\partial \mu}=\Sigma^{-1}(I(x)-\mu)
$$


We can compute the gradient with respect to the covariance matrix $\Sigma$ in the same manner:

$$
\frac{\partial}{\partial \Sigma} G=\Sigma^{-1}\left(\Sigma-(I-\mu)(I-\mu)^{T}\right) \Sigma^{-1}
$$

The second term of the first variation of $F$ can be simplified:

$$
\left.\int_{\Omega} \frac{\partial G(x, \phi+\epsilon \psi)}{\partial \epsilon}\right|_{\epsilon=0} \chi(\phi) d x=\left.\int_{\Omega} \frac{\partial G}{\partial \mu} \frac{\partial \mu}{\partial \epsilon}\right|_{\epsilon=0} \chi(\phi) d x+\left.\int_{\Omega} \frac{\partial G}{\partial \Sigma} \frac{\partial \Sigma}{\partial \epsilon}\right|_{\epsilon=0} \chi(\phi) d x
$$

and we can compute the two integrals over $\Omega$ :

$$
\begin{aligned}
\int_{\Omega} \frac{\partial G}{\partial \mu} \chi(\phi) d x & =\int_{\Omega} \Sigma^{-1}(I(x)-\mu) \chi(\phi) d x \\
& =\Sigma^{-1}\left(\int_{\Omega} I(x) \chi(\phi) d x-\mu \int_{\Omega} \chi(\phi) d x\right)=0
\end{aligned}
$$

and

$$
\begin{aligned}
\int_{\Omega} \frac{\partial G}{\partial \Sigma} \chi(\phi) \mathrm{d} x & =\int_{\Omega} \Sigma^{-1}\left(\Sigma-(I(x)-\mu)(I(x)-\mu)^{T}\right) \Sigma^{-1} \chi(\phi) \mathrm{d} x \\
& =\Sigma^{-1}\left(\Sigma \int_{\Omega} \chi(\phi) \mathrm{d} x-\int_{\Omega}(I(x)-\mu)(I(x)-\mu)^{T} \chi(\phi) \mathrm{d} x\right) \Sigma^{-1} \\
& =0
\end{aligned}
$$

It follows that the second term is null. The first variation of $\mathrm{F}$ can simply be expressed as:

$$
\left.\frac{\partial F(\phi+\epsilon \psi)}{\partial \epsilon}\right|_{\epsilon=0}=\frac{1}{2} \int_{\Omega} \log p(I(x) \mid \mu, \Sigma) \chi^{\prime}(\phi) d x
$$

\section{A.2 Shape derivative method}

In this subsection, we propose to use the shape derivative tool introduced in [1] in order to derive the following functional depending on the domain $\Omega$ :

$$
\begin{aligned}
D(\Omega) & =\int_{\Omega} k(x, \Omega) d x \\
& =\int_{\Omega}\left(\log |\Sigma(\Omega)|+(I(x)-\mu(\Omega))^{T} \Sigma(\Omega)^{-1}(I(x)-\mu(\Omega))\right) d x
\end{aligned}
$$

RR $\mathrm{n}^{\circ} 4515$ 
with:

$$
\left\{\begin{array}{l}
\mu(\Omega)=\frac{1}{V(\Omega)} \int_{\Omega} I(x) d x \\
S(\Omega)=\frac{1}{V(\Omega)} \int_{\Omega}(I(x)-\mu(\Omega))(I(x)-\mu(\Omega))^{T} d x \\
V(\Omega)=\int_{\Omega} d x
\end{array}\right.
$$

Following [1], we compute the Gâteaux derivative of $D(\Omega)$ in the direction of $V$ :

$$
<D^{\prime}(\Omega), V>=\int_{\Omega} k_{s}(x, \Omega, V) d x-\int_{\Gamma} k(x, \Omega)(V(x) \cdot N(x)) d a(x)
$$

where $k_{s}(x, \Omega, V)$ is the shape derivative of $k(x, \Omega)$.

$$
\begin{aligned}
k_{s}(x, \Omega, V) & =f_{s}(x, \mu(\Omega), \Sigma(\Omega), V) \\
& =f_{\mu}<\mu^{\prime}(\Omega), V>+f_{\Sigma}<\Sigma^{\prime}(\Omega), V>
\end{aligned}
$$

The derivations of $f$ with respect to $\mu$ and $\Sigma$ give:

$$
\left\{\begin{array}{l}
f_{\mu}=\Sigma^{-1}(I(x)-\mu) \\
f_{\Sigma}=\Sigma^{-1}\left(\Sigma-(I-\mu)(I-\mu)^{T}\right) \Sigma^{-1}
\end{array}\right.
$$

Since $\mu(\Omega)$ and $S(\Omega)$ do not depend on $x$, we have:

$$
\int_{\Omega} f_{s}(x, \ldots, V) d x=<\mu^{\prime}(\Omega), V>\int_{\Omega} f_{\mu}(x, \ldots) d x+<\Sigma^{\prime}(\Omega), V>\int_{\Omega} f_{\Sigma}(x, \ldots) d x
$$

Similarly to the first method, both integrals over $\Omega$ of $f_{\mu}$ and $f_{\Sigma}$ are equal to zero. Then, only the second term of the derivative has to be considered:

$$
<D^{\prime}(\Omega), V>=-\int_{\Gamma} k(x, \Omega)(V(x) \cdot N(x)) d a(x)
$$

\section{References}

[1] G. Aubert, M. Barlaud, O. Faugeras, and S. Jehan-Besson. Image segmentation using active contours: calculus of variations of shape gradients? Research Report, INRIA, July 2002.

[2] T. Chan, B.Y. Sandberg, and L. Vese. Active contours without edges for vectorvalued images. Journal of Visual Communication and Image Representation, 11:130-141, 2000. 
[3] D. Cremers, T. Kohlberger, and C. Schnörr. Nonlinear shape statistics in mumford-shah based segmentation. In European Conf. on Computer Vision, volume 2, pages 93-108, Copenhagen, Denmark, June 2002.

[4] S. Jehan-Besson, M. Barlaud, and G. Aubert. Dream2s: Deformable regions driven by an eulerian accurate minimization method for image and video segmentation, application to face detection in color video sequences. In $E C C V$, volume 3, pages 365-380, Copenhagen, Denmark, May 2002.

[5] Junmo Kim, John W. Fisher III, Mujdat Cetin Anthony Yezzi, Jr. and, and Alan S. Willsky. Nonparametric methods for image segmentation using information theory and curve evolution. In IEEE International Conference on Image Processing, Rochester, NY, September 2002.

[6] Y.G. Leclerc. Constructing simple stable description for image partitioning. The International Journal of Computer Vision, 3(1):73-102, 1989.

[7] Michael Leventon, Olivier Faugeras, Eric Grimson, and William Wells. Level set based segmentation with intensity and curvature priors. In Mathematical Methods in Biomedical Image Analysis, South Carolina, 2000.

[8] Michael Leventon, Eric Grimson, and Olivier Faugeras. Statistical shape influence in geodesic active contours. In CVPR, pages 316-323, 2000.

[9] Nikos Paragios and Rachid Deriche. Geodesic active contours and level set methods for supervised texture segmentation. International Journal of Computer Vision, 46(3):223-247, March 2002.

[10] Nikos Paragios and Rachid Deriche. Geodesic active regions: A new paradigm to deal with frame partition problems in computer vision. Journal of Visual Communication and Image Representation, March/June 2002.

[11] Mikael Rousson and Nikos Paragios. Shape priors for level set representations. In $E C C V$, volume 2, pages 78-92, Copenhagen, Denmark, May 2002.

[12] J. Weickert, B.M. ter Haar Romeny, and M.A. Viergever. Efficient and reliable schemes for nonlinear diffusion filtering. IEEE Transactions on Image Processing, 7(3):398-410, March 1998.

[13] A. Yezzi, A. Tsai, and A. Willsky. A statistical approach to snakes for bimodal and trimodal imagery. In Proceedings of the 7th International Conference on

$\mathrm{RR} \mathrm{n}^{\circ} 4515$ 
Computer Vision, volume II, pages 898-903, Kerkyra, Greece, September 1999. IEEE Computer Society, IEEE Computer Society Press.

[14] S. Zhu and A. Yuille. Region competition: unifying snakes, region growing, and Bayes/MDL for multiband image segmentation. IEEE Transactions on Pattern Analysis and Machine Intelligence, 18(9):884-900, September 1996. 


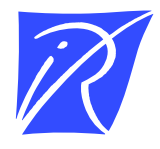

Unité de recherche INRIA Sophia Antipolis 2004, route des Lucioles - BP 93 - 06902 Sophia Antipolis Cedex (France)

Unité de recherche INRIA Lorraine : LORIA, Technopôle de Nancy-Brabois - Campus scientifique 615, rue du Jardin Botanique - BP 101 - 54602 Villers-lès-Nancy Cedex (France)

Unité de recherche INRIA Rennes : IRISA, Campus universitaire de Beaulieu - 35042 Rennes Cedex (France)

Unité de recherche INRIA Rhône-Alpes : 655, avenue de l'Europe - 38330 Montbonnot-St-Martin (France)

Unité de recherche INRIA Rocquencourt : Domaine de Voluceau - Rocquencourt - BP 105 - 78153 Le Chesnay Cedex (France)

Éditeur

INRIA - Domaine de Voluceau - Rocquencourt, BP 105 - 78153 Le Chesnay Cedex (France)

http://www.inria.fr

ISSN 0249-6399 